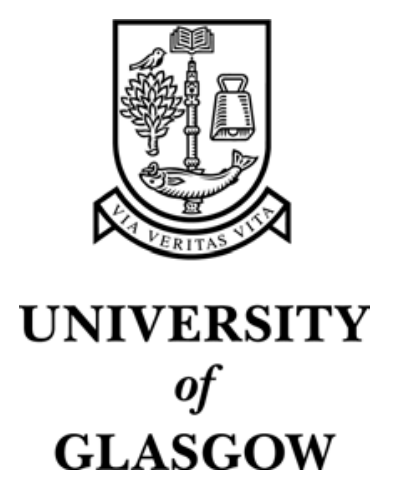

Iwama, K. and Manlove, D. and Miyazaki, S. and Morita, Y. (1999)

Stable marriage with ties and incomplete lists. In, Wiedermann, J. and

Van Emde Boas, P. and Nielsen, M., Eds. Proceedings of ICALP '99: the 26th International Colloquium on Automata, Languages and

Programming, 11-15 July, 1999 Lecture Notes in Computer Science Vol 1644, pages 443-452, Prague, Czech Republic.

http://eprints.gla.ac.uk/archive/00001066/ 


\title{
Stable Marriage with Incomplete Lists and Ties
}

\author{
Kazuo Iwama $^{1 \star}$, David Manlove ${ }^{2 \star \star}$, Shuichi Miyazaki ${ }^{1}$ and Yasufumi Morita ${ }^{1}$ \\ 1 School of Informatics, Kyoto University, Kyoto 606-8501, Japan \\ \{iwama, shuichi, ymorita\}@kuis.kyoto-u.ac.jp \\ 2 Dept. of Computing Science, University of Glasgow, Glasgow G12 8QQ, Scotland \\ davidm@dcs.gla.ac.uk
}

\begin{abstract}
The original stable marriage problem requires all men and women to submit a complete and strictly ordered preference list. This is obviously often unrealistic in practice, and several relaxations have been proposed, including the following two common ones: one is to allow an incomplete list, i.e., a man is permitted to accept only a subset of the women and vice versa. The other is to allow a preference list including ties. Fortunately, it is known that both relaxed problems can still be solved in polynomial time. In this paper, we show that the situation changes substantially if we allow both relaxations (incomplete lists and ties) at the same time: the problem not only becomes NP-hard, but also the optimal cost version has no approximation algorithm achieving the approximation ratio of $N^{1-\epsilon}$, where $N$ is the instance size, unless $\mathrm{P}=\mathrm{NP}$.
\end{abstract}

\section{Introduction}

An instance of the stable marriage problem [4] consists of $N$ men and $N$ women. Each person has his/her strictly ordered preference list containing all the members of the opposite sex. A matching $M$ is a one-one correspondence between all the men and all the women. If a man $m$ and a woman $w$ are matched in $M$, we say $m$ and $w$ are partners in $M$, and we write $M(m)=w$ and $M(w)=m$. A man $m$ and a woman $w$ form a blocking pair for a matching $M$ if $m$ and $w$ are not partners in $M$, but $m$ prefers $w$ to $M(m)$ and $w$ prefers $m$ to $M(w)$. If there is no blocking pair for $M$, then $M$ is called stable. The stable marriage problem was first studied by Gale and Shapley [1]. They showed that there always exists at least one stable matching in any instance and gave an $O\left(N^{2}\right)$-time algorithm, the so-called Gale-Shapley algorithm, to find one.

Since then, this problem has been constantly one of the most popular combinatorial problems from both theoretical and practical view points [e.g., 4]. Considering practical applications, however, the above restrictions for preference lists, namely total order and completeness, appear to be too strict on many occasions, and therefore some extensions of this problem have been proposed. The popular ones are (i) the stable marriage problem with unacceptable partners [4], and (ii) the stable marriage problem with indifference [4,6]. In the first extension, each person is allowed to declare one or more unacceptable partners. Thus each person's preference list may be incomplete. Gale and Sotomayor [2]

\footnotetext{
* Supported in part by Scientific Research Grant, Ministry of Japan, 10558044, 09480055 and 10205215.

** Supported by Engineering and Physical Sciences Research Council grant number GR/M13329.
} 
studied this extension and showed that there is a polynomial-time algorithm which determines whether there exists a stable matching and finds one if one exists (see also [4]). Thus the problem does not become essentially harder. (In this paper, the term "matching" when incomplete lists are allowed refers to a one-one correspondence between all the men and all the women, as is the definition for complete lists.)

In the second extension, each person is allowed to have a preference list with ties. $m$ and $w$ form a blocking pair if $m$ and $w$ are not partners in $M$, but $m$ strictly prefers $w$ to $M(m)$ (i.e., $w$ and $M(m)$ are not tied in $m$ 's list) and $w$ strictly prefers $m$ to $M(w)$. A matching containing no such blocking pair is called weakly stable. (Henceforth, usage of the term "a stable matching" when ties are allowed in the lists refers to weak stability.) However, this extension also does not make the problem significantly difficult; it is known that there always exists a stable matching (and a polynomial-time algorithm finds one) [4].

Now arises the natural question, i.e., whether or not the situation changes if we apply both extensions at the same time, which was open since [6]. Our main purpose of this paper is to show that the situation does change, i.e., the problem is now NP-complete if we allow both incomplete lists and ties. The problem is also intractable for complete lists if the question is not the existence of stable matchings but obtaining optimal cost stable matchings. We furthermore show that it is probably not possible to obtain good approximation algorithms for the optimal cost problem: unless $\mathrm{P}=\mathrm{NP}$, any approximation algorithm cannot achieve $N^{1-\epsilon}$ for any $\epsilon>0$ as its approximation ratio.

The general perception for the stable marriage problem has been that it is basically not hard $[1,4,6]$. Our results could alter this common perception. (In the case of non-bipartite setting, i.e., for the so-called hospitals/residents problem and the stable roommates problem, some intractable results have been reported [8], but none previously for the bipartite setting.)

\section{Stable Marriage Problems}

Recall that the original stable matching problem requires each person's preference list to be complete (i.e., all the members of the opposite sex must be included) and to be totally ordered. We focus on the three possible relaxations concerning these two restrictions. All these problems (including the original one) ask if there exists a stable matching. The first three problems are known to be in $\mathrm{P}[2,4]$. Our main result is the NP-completeness of the last one, shown in Sec.3.

SMP-CLTO: the stable marriage problem with complete list and total order. SMP-ILTO: the stable marriage problem with incomplete list and total order. SMP-CLT: the stable marriage problem with complete list and ties.

SMP-ILT: the stable marriage problem with incomplete list and ties.

If we consider the cost of a matching, we can introduce an optimization version of the stable marriage problem. Suppose that, under a stable matching $M$, a man $m$ is matched with a woman $w$ who is at the $i$ th position in $m$ 's list. Then it is defined that the cost of the position of woman $w$ in $m$ 's list is $i$ and the cost of the matching $M$ for man $m$ is also $i$. The cost for a woman $w$ is similarly defined. If there are ties in the list, then the cost of positions are determined as usual: for example, if $m$ 's list is $w_{3},\left(w_{2}, w_{4}, w_{5}\right), w_{1}$, where $w_{2}, w_{4}$ and $w_{5}$ are tied in the list, then the cost of the positions for $w_{3}, w_{2}, w_{4}, w_{5}$ and $w_{1}$ are 1 , 2, 2, 2 and 5, respectively. The cost of the matching $M$ is the sum of the costs of $M$ for all $2 N$ people. (The stable matching that minimizes this cost is called 
the egalitarian stable matching [4].) Here we consider the following two problems whose preference lists are always complete:

MIN-SMP: Preference lists must be a total order.

MIN-SMP-TIES: Preference lists may contain ties.

Both problems ask to find a stable matching whose cost is as small as possible. It is known that an optimal solution for MIN-SMP may be obtained in polynomial time [4]. We shall show the NP-hardness and inapproximability of MIN-SMP-TIES in Sec.4.

\section{Intractability Results}

Now we prove the NP-completeness of SMP-ILT. Note that this proof is further extended to show the inapproximability of MIN-SMP-TIES in the next section.

Theorem 1. SMP-ILT is NP-complete.

Proof. It is easy to see that SMP-ILT is in NP: given a matching $M$, one can check, for each man $m$ and woman $w$, whether $m$ and $w$ form a blocking pair. This can be done in polynomial time. To show the NP-hardness, let us consider the problem ONE-IN-THREE 3SAT: given a 3CNF formula, it asks if there exists a truth assignment such that exactly one literal in each clause is true.

It is known that ONE-IN-THREE 3SAT remains NP-complete even if a $3 \mathrm{CNF}$ formula does not include negative literals [3]. So, we translate this restricted problem into SMP-ILT. Given $f$, which is an instance of ONE-INTHREE 3SAT, we construct an instance $T(f)$ of SMP-ILT consisting of (1) a set of men and the same number of women, $(2)$ each man's preference list, and (3) each woman's preference list. Let $n$ and $l$ be the numbers of variables and clauses of $f=C_{1} \cdot C_{2} \cdots C_{l}$, respectively. Let $t_{i}$ be the number of appearances of the variable $x_{i}$ and let $t=\max \left\{t_{1}, t_{2}, \cdots, t_{n}\right\}$.

\subsection{The set of Men and Women}

$T(f)$ consists of $9 l+3 n+t+3$ men and the same number of women. We first introduce the following set of men, who are divided into the following groups:

Group (A): $t+3$ men $m_{A, 1}, \cdots, m_{A, t+3}$. Necessary for a technical reason.

Group (B): $3 l$ men $m_{B, i, j}$ for $1 \leq i \leq l$ and $1 \leq j \leq 3$. The three men $m_{B, i, 1}$, $m_{B, i, 2}$ and $m_{B, i, 3}$ correspond to the clause $\bar{C}_{i}$.

Group (C): $n$ men $m_{C, 1}, \cdots, m_{C, n} . m_{C, i}$ is associated with the variable $x_{i}$.

Group (D): $2 n$ men $m_{D, 1}^{+}, m_{D, 1}^{-}, \cdots, m_{D, n}^{+}, m_{D, n}^{-} \cdot m_{D, i}^{+}$and $m_{D, i}^{-}$are associated with the variable $x_{i}$. (Note that these + and - signs do not relate to the polarity or the true/false value of $x_{i}$.)

Group (E): $6 l$ men $m_{E, i, j}^{+}, m_{E, i, j}^{-}$. For each $1 \leq i \leq n$ and $1 \leq j \leq l, m_{E, i, j}^{+}$and $m_{E, i, j}^{-}$exist if and only if the literal $x_{i}$ appears in the clause $C_{j}$. (Recall that $\overline{x_{i}}$ never appears in $f$.) Since there are $3 l$ literals, $6 l$ men will be introduced.

The same number $(9 l+3 n+t+3)$ of women are also divided into the following five groups:

Group (a): $t+3$ women $w_{a, 1}, \cdots, w_{a, t+3}$ as Group (A) of the men.

Group (b): $2 n$ women $w_{b, i}^{0}$ and $w_{b, i}^{1}(1 \leq i \leq n)$. Two women $w_{b, i}^{0}$ and $w_{b, i}^{1}$ are associated with the variable $x_{i}$. 
Group (c): $n$ women $w_{c, i}(1 \leq i \leq n) . w_{c, i}$ is associated with the variable $x_{i}$. Group (d): $6 l$ women $w_{d, i, j}^{0}$ and $w_{d, i, j}^{1}$. For $1 \leq i \leq n$ and $1 \leq j \leq l$, two women $w_{d, i, j}^{0}$ and $w_{d, i, j}^{1}$ exist if and only if the literal $x_{i}$ appears in the clause $C_{j}$.

Group (e): $3 l$ women $w_{e, i, j}$. Similarly as Group-(d) women, a woman $w_{e, i, j}$ exists if and only if the literal $x_{i}$ appears in the clause $C_{j}$.

\subsection{Men's Preference Lists}

We then construct each man's preference list (obviously in polynomial time). For better exposition, we use an example of $f$, i.e., $f_{0}=\left(x_{1}+x_{2}+x_{3}\right)\left(x_{1}+x_{3}+x_{4}\right)$, for which the men's preference lists will turn out to be as illustrated in Table 2 . As for each Group-(A) man $m_{A, i}$, his list only includes the single woman $w_{a, i}$.

Recall that the three men $m_{B, i, 1}, m_{B, i, 2}$ and $m_{B, i, 3}$ in Group (B) are associated with the clause $C_{i}$. We show how to construct preference lists of men $m_{B, 2,1}, m_{B, 2,2}$ and $m_{B, 2,3}$ who are associated with $C_{2}=\left(x_{1}+x_{3}+x_{4}\right)$ of $f_{0}$. Since literals $x_{1}, x_{3}$ and $x_{4}$ appear in $C_{2}$, six women $w_{d, 1,2}^{0}, w_{d, 1,2}^{1}$ (associated with $x_{1}$ in $\left.C_{2}\right), w_{d, 3,2}^{0}, w_{d, 3,2}^{1}$ (associated with $x_{3}$ in $C_{2}$ ), and $w_{d, 4,2}^{0}, w_{d, 4,2}^{1}$ (associated with $x_{4}$ in $C_{2}$ ) have been introduced. $m_{B, 2,1}$ writes $w_{d, 1,2}^{1}, w_{d, 3,2}^{1}$ and $w_{d, 4,2}^{1}$ at the first position. (These three women are tied in the list.) Both $m_{B, 2,2}$ and $m_{B, 2,3}$ write $w_{d, 1,2}^{0}, w_{d, 3,2}^{0}$ and $w_{d, 4,2}^{0}$ at the first position. Intuitively speaking, the man $m_{B, i, 1}$ will be matched with the woman who corresponds to the literal having the value 1 , and men $m_{B, i, 2}$ and $m_{B, i, 3}$ will be matched with the women who correspond to literals having the value 0 .

Each man in Group (C) selects $t+5$ women at the 1 st through $(t+5)$ th positions of the preference lists. The women at the 1st through $(t+3)$ th positions are $w_{a, 1}$ through $w_{a, t+3}$, in this order. $m_{C, i}$ writes the woman $w_{b, i}^{0}$ at the $(t+4)$ th position and the woman $w_{b, i}^{1}$ at the $(t+5)$ th position. Intuitively speaking, assigning a man $m_{C, i}$ to the woman $w_{b, i}^{0}$ (respectively $w_{b, i}^{1}$ ) means assigning 0 (respectively 1) to the variable $x_{i}$.

Then we construct preference lists of Group-(D) men. We show how to construct preference lists using men $m_{D, 1}^{+}$and $m_{D, 1}^{-}$for $f_{0}$. (Again, see Table 2.) Recall that these two men are associated with the variable $x_{1}$. The man $m_{D, 1}^{+}$ writes the woman $w_{c, 1}$ at the 2 nd position. (The 2 nd position is always determined without depending on $f$.) Then, $m_{D, 1}^{+}$writes the woman $w_{b, 1}^{0}$ at the $t+3(=5)$ th position. Since $x_{1}$ appears in clauses $C_{1}$ and $C_{2}$, two women $w_{d, 1,1}^{1}$ and $w_{d, 1,2}^{1}$ have been introduced. $m_{D, 1}^{+}$writes $w_{d, 1,1}^{1}$ and $w_{d, 1,2}^{1}$ at the 3 rd and 4 th positions, respectively. The other positions are filled with (some of) $w_{a, 1}$ through $w_{a, 5} ; m_{D, 1}^{+}$writes $w_{a, i}$ at the $i$ th position if the $i$ th position is blank. Generally speaking, there are $t_{i}$ women of the form $w_{d, i, j}^{1}$ corresponding to the variable $x_{i}$. (Recall that $t_{i}$ is the number of appearances of the literal $x_{i}$.) $m_{D, i}^{+}$writes these women at $3 \mathrm{rd}$ through $\left(t_{i}+2\right)$ th positions. Since $t_{i} \leq t$, these women's positions never go to the $(t+3)$ th position which is already occupied by $w_{b, i}^{0}$.

$m_{D, 1}^{-}$'s list is similarly constructed. $m_{D, 1}^{-}$writes the woman $w_{c, 1}$ at the 1 st position and writes the woman $w_{b, 1}^{1}$ at the $t+3(=5)$ th position. There are two women $w_{d, 1,1}^{0}$ and $w_{d, 1,2}^{0}$ associated with the variable $x_{1}$ since $x_{1}$ appears in $C_{1}$ 
and $C_{2} \cdot m_{D, 1}^{-}$writes $w_{d, 1,1}^{0}$ and $w_{d, 1,2}^{0}$ at the 3rd and 4 th positions, respectively. Blanks are filled as above.

Now we move to Group-(E) men: the man $m_{E, 1,2}^{+}$, associated with $x_{1}$ in $C_{2}$, writes the woman $w_{e, 1,2}$ at the 2 nd position and writes $w_{d, 1,2}^{1}$ at the same position as $m_{D, 1}^{+}$(associated with $x_{1}$ ) wrote it. $m_{E, 1,2}^{-}$writes $w_{e, 1,2}$ at the 1 st position and writes $w_{d, 1,2}^{0}$ at the same position as $m_{D, 1}^{-}$wrote it. Blanks are filled with women $w_{a, 1}$ through $w_{a, 5}$ similarly as the Group-(D) men did. Now the men's lists are completed. Table 2 shows the whole lists of men of $T\left(f_{0}\right)$.

\subsection{Women's Preference Lists}

Finally, we construct the women's preference lists. We construct the women's preference lists automatically from the men's preference lists. First, we determine the total order of all men; the position of each man in the order is called his rank. The rank of each man of our current example $T\left(f_{0}\right)$ is shown in Table 2 , e.g., $m_{A, 1}$ is the highest and $m_{E, 4,2}^{-}$is the lowest. Generally speaking, the men are lexicographically ordered, where the significance of the indices of $m_{\alpha, \beta, \gamma}^{\delta}$ is in the order of $\alpha, \beta, \gamma$ and $\delta$, e.g., $\alpha$ is the most significant index and $\delta$ is the least significant index. For $\alpha$, the priority is given to $A, B, C, D$ and $E$ in this order. For $\beta$ and $\gamma$, the smaller number precedes the larger number. For $\delta$, precedes -.

Women's lists are constructed based on this order. First of all, the preference list of a woman $w$ does not include a man $m$ if $w$ does not appear on $m$ 's preference list. Consider two men $m_{i}$ and $m_{j}$ included in $w$ 's list. $w$ strictly prefers $m_{i}$ to $m_{j}$ if and only if (1) the rank of $m_{i}$ is higher than that of $m_{j}$, and (2) the position of $w$ in $m_{i}$ 's list is higher than or equal to the position of $w$ in $m_{j}$ 's list. One might think that a woman's list can contain a partial order in this construction. However, in our translation, each woman's list contains only ties.

It helps much to know that, by our construction of the women's preference lists, we can determine whether a matching includes a blocking pair or not from only the men's preference lists. Consider two men $m_{i}$ and $m_{j}$ who are matched with $w_{i}$ and $w_{j}$, respectively. Then, $\left(m_{i}, w_{j}\right)$ is a blocking pair if and only if (i) $m_{i}$ strictly prefers $w_{j}$ to $w_{i}$, (ii) $m_{i}$ 's rank is higher than $m_{j}$ 's rank, and (iii) the position of $w_{j}$ in $m_{i}$ 's list is higher than or equal to the position of $w_{j}$ in $m_{j}$ 's list. Observe that the combination of conditions (ii) and (iii) means that $w_{j}$ strictly prefers $m_{i}$ to $m_{j}$.

\subsection{Useful Lemmas}

Now the translation is completed. Next, we show a series of lemmas which summarize several conditions for a matching $M$ for $T(f)$ to be a solution. Proofs are not so difficult and are omitted. Recall that we write $M(m)=w$ if a man $m$ and a woman $w$ are matched in $M$.

Lemma 1. If a matching $M$ for $T(f)$ is a solution, then $M\left(m_{A, i}\right)=w_{a, i}$ $(1 \leq i \leq t+3)$. Namely, each man in Group (A) is matched with the woman at the first position on his list.

Lemma 2. If a matching $M$ for $T(f)$ is a solution, then for each $i(1 \leq i \leq n)$, $M\left(m_{C, i}\right)=w_{b, i}^{0}$ or $M\left(m_{C, i}\right)=w_{b, i}^{1}$.

Lemma 3. Suppose that a matching $M$ for $T(f)$ is a solution. Then for all $1 \leq i \leq n$, (i) if $M\left(m_{C, i}\right)=w_{b, i}^{0}$ then $M\left(m_{D, i}^{+}\right)=w_{c, i}$ and $M\left(m_{D, i}^{-}\right)=w_{b, i}^{1}$. (ii) Otherwise, i.e., if $M\left(m_{C, i}\right)=w_{b, i}^{1}$, then $M\left(m_{D, i}^{+}\right)=w_{b, i}^{0}$ and $M\left(m_{D, i}^{-}\right)=w_{c, i}$. 
Lemma 4. Suppose that a matching $M$ for $T(f)$ is a solution. Then the following statements (i) and (ii) are true for all $i(1 \leq i \leq n)$. (i) If $M\left(m_{C, i}\right)=$ $w_{b, i}^{0}$ then $M\left(m_{E, i, j}^{+}\right)=w_{d, i, j}^{1}$ and $M\left(m_{E, i, j}^{-}\right)=w_{e, i, j}$ for all $j$. (ii) If $M\left(m_{C, i}\right)=$ $w_{b, i}^{1}$ then $M\left(m_{E, i, j}^{+}\right)=w_{e, i, j}$ and $M\left(m_{E, i, j}^{-}\right)=w_{d, i, j}^{0}$ for all $j$.

Then we go back to Group-(B) men. Let $C_{j}=\left(x_{j_{1}}+x_{j_{2}}+x_{j_{3}}\right)$ be the $j$ th clause in $f$. Then recall that there are three men $m_{B, j, 1}, m_{B, j, 2}$ and $m_{B, j, 3}$, and six women $w_{d, j_{1}, j}^{0}, w_{d, j_{1}, j}^{1}, w_{d, j_{2}, j}^{0}, w_{d, j_{2}, j}^{1}, w_{d, j_{3}, j}^{0}$ and $w_{d, j_{3}, j}^{1}$ (see Table 1$)$.

\begin{tabular}{|l||lll|}
\hline$m_{B, j, 1}$ & $w_{d, j_{1}, j}^{1}$ & $w_{d, j_{2}, j}^{1}$ & $w_{d, j_{3}, j}^{1}$ \\
\hline$m_{B, j, 2}$ & $w_{d, j_{1}, j}^{0}$ & $w_{d, j_{2}, j}^{0}$ & $w_{d, j_{3}, j}^{0}$ \\
\hline$m_{B, j, 3}$ & $w_{d, j_{1}, j}^{0}$ & $w_{d, j_{2}, j}^{0}$ & $w_{d, j_{3}, j}^{0}$ \\
\hline
\end{tabular}

Table 1. Preference lists of men associated to $C_{j}$

Lemma 5. Suppose that a matching $M$ for $T(f)$ is a solution. Then, for any $i \in\left\{j_{1}, j_{2}, j_{3}\right\}$, if $w_{d, i, j}^{1}$ is matched with $m_{B, j, 1}$, then $w_{d, i, j}^{0}$ is not matched with any of $m_{B, j, 2}$ and $m_{B, j, 3}$. Namely, for any $i$ and $j$, one of $w_{d, i, j}^{0}$ and $w_{d, i, j}^{1}$ is matched with a man in Group (B) and the other is matched with a man not in Group (B).

Lemma 6. Suppose that a matching $M$ for $T(f)$ is a solution. Then, for each $i$ and $j$, the following statements (i), (ii) and (iii) are true: (i) If $M\left(m_{B, j, 1}\right)=w_{d, i, j}^{1}$ then $M\left(m_{C, i}\right)=w_{b, i}^{1}$. (ii) If $M\left(m_{B, j, 2}\right)=w_{d, i, j}^{0}$ then $M\left(m_{C, i}\right)=w_{b, i}^{0}$. (iii) If $M\left(m_{B, j, 3}\right)=w_{d, i, j}^{0}$ then $M\left(m_{C, i}\right)=w_{b, i}^{0}$.

\subsection{Correctness of the Reduction}

Now we are ready to show the correctness of the reduction. To make the argument clear, we denote the literal $x_{i}$ in the clause $C_{j}$ by $x_{i}^{j}$ and regard $x_{i}^{j}$ as a different object from the variable $x_{i}$. Let us consider the following association rule between an assignment for variables (and literals) of $f$ and a matching $M$ for $T(f)$ : (i) assign 1 to the variable $x_{i}$ if and only if the man $m_{C, i}$ and the woman $w_{b, i}^{1}$ are matched in $M$, and assign 0 to the variable $x_{i}$ if and only if the man $m_{C, i}$ and the woman $w_{b, i}^{0}$ are matched in $M$. (ii) Assign 1 to the literal $x_{i}^{j}$ if and only if the woman $w_{d, i, j}^{1}$ is matched with the man $m_{B, j, 1}$ in $M$, and assign 0 to the literal $x_{i}^{j}$ if and only if the woman $w_{d, i, j}^{0}$ is matched with the man $m_{B, j, 2}$ or $m_{B, j, 3}$.

Lemma 7. The association rule is consistent, namely, if $M$ is a solution for $T(f)$, then the following two statements hold: (i) if $x_{i}=1$ then $x_{i}^{j}=1$ for all $j$. (ii) If $x_{i}=0$ then $x_{i}^{j}=0$ for all $j$. (Proof is omitted.)

Now suppose that there exists a solution $M^{*}$ for $T(f)$. Then we can show that there exists a solution for $f$ by the following four steps: (1) first, we determine the value of each variable of $f$. By Lemma 2 , either $M^{*}\left(m_{C, i}\right)=w_{b, i}^{0}$ or $M^{*}\left(m_{C, i}\right)=$ $w_{b, i}^{1}$ for all $i$. Due to this, we determine the assignment using the association rule: if $M^{*}\left(m_{C, i}\right)=w_{b, i}^{0}$ then $x_{i}=0$, otherwise, i.e., if $M^{*}\left(m_{C, i}\right)=w_{b, i}^{1}$, then $x_{i}=1$. (2) Again we use the association rule to determine the value of literals: if $M^{*}\left(w_{d, i, j}^{1}\right)=m_{B, j, 1}$, then $x_{i}^{j}=1$. If $M^{*}\left(w_{d, i, j}^{0}\right)=m_{B, j, 2}$ or $M^{*}\left(w_{d, i, j}^{0}\right)=m_{B, j, 3}$, then $x_{i}^{j}=0$. It should be noted, by Lemma 5 , that only one value is assigned to each literal $x_{i}^{j}$. (3) Let, for $1 \leq j \leq l$, the $j$ th clause of $f$ be $C_{j}=\left(x_{j_{1}}+x_{j_{2}}+\right.$ 
$x_{j_{3}}$ ). Then the preference lists of the three men associated to $C_{j}$ are the ones described in Table 1 . Since $M^{*}$ is a solution, these men must be matched in the way described in Lemma 5. There are six different possibilities. Suppose that $M^{*}\left(m_{B, j, 1}\right)=w_{d, j_{1}, j}^{1}, M^{*}\left(m_{B, j, 2}\right)=w_{d, j_{2}, j}^{0}$ and $M^{*}\left(m_{B, j, 3}\right)=w_{d, j_{3}, j}^{0}$. Then, by the assignment determined in (2) above, we have $x_{j_{1}}^{j}=1, x_{j_{2}}^{j}=0$ and $x_{j_{3}}^{j}=0$, namely, exactly one literal in $C_{j}$ is true. It is not hard to see that, in the other five cases also, exactly one literal in $C_{j}$ is true. (4) By Lemma 7, this assignment is actually a solution of $f$.

Conversely, suppose that there exists a solution for $f$ of ONE-IN-THREE 3SAT. Then, again using the association rule, we determine partners for the men in Groups (B) and (C). Partners for the men in Groups (A), (D) and (E) are automatically determined using Lemmas 1,3 and 4 . The fact that this matching is a solution for $T(f)$ can be easily seen from Lemmas 1 through 5 .

\subsection{An Alternative Proof of Theorem 1}

An alternative, shorter proof of the NP-completeness of SMP-ILT may be obtained by transforming from the problem EXACT MAXIMAL MATCHING, which takes a graph $G$ and an integer $K$ as input, and asks whether $G$ has a maximal matching $M$ with $|M|=K$. EXACT MAXIMAL MATCHING is NPcomplete, even for bipartite graphs - this follows from the NP-completeness of the minimization version, MINIMUM MAXIMAL MATCHING, for the same class of graphs [9].

Proof. Membership of SMP-ILT in NP was established in Theorem 1. To show the NP-hardness of SMP-ILT, we transform from EXACT MAXIMAL MATCHING for bipartite graphs; let $G=(V, E)$ and $K$ be an instance of this problem. Then $G$ has a bipartition $\langle U, W\rangle$. Without loss of generality we may assume that $|U|=|W|=n$, and that $K \leq n$. Let $U=\left\{m_{1}, m_{2}, \ldots, m_{n}\right\}$ and $W=\left\{w_{1}, w_{2}, \ldots, w_{n}\right\}$. We construct an instance $I$ of SMP-ILT as follows: let $U \cup X$ be the set of men, and let $W \cup Y$ be the set of women, where $X=$ $\left\{x_{1}, x_{2}, \ldots, x_{n-K}\right\}$ and $Y=\left\{y_{1}, y_{2}, \ldots, y_{n-K}\right\}$. For any $m_{i}(1 \leq i \leq n)$, let $M_{i}$ contain the women $w_{j}$ such that $\left\{m_{i}, w_{j}\right\} \in E$. For any $w_{j}(1 \leq j \leq n)$, let $W_{j}$ contain the men $m_{i}$ such that $\left\{m_{i}, w_{j}\right\} \in E$. Create preference lists for each person as follows:

$$
\begin{array}{ccl}
m_{i}:\left(\text { members of } M_{i}\right)\left(y_{1} \ldots y_{n-K}\right) & (1 \leq i \leq n) \\
x_{i}: & \left(w_{1} \ldots w_{n}\right) & (1 \leq i \leq n-K) \\
w_{j}:\left(\text { members of } W_{j}\right)\left(x_{1} \ldots x_{n-K}\right) & (1 \leq j \leq n) \\
y_{j}: & \left(m_{1} \ldots m_{n}\right) & (1 \leq j \leq n-K)
\end{array}
$$

In a preference list, persons within parentheses are tied. We claim that $G$ has a maximal matching of size exactly $K$ if and only if $I$ has a stable matching.

For, suppose that $G$ has a maximal matching $M$ where $|M|=K$. We construct a matching $M^{\prime}$ in $I$ as follows. For each edge $\left\{m_{i}, w_{j}\right\}$ in $M$, we let $w_{j}$ be the partner of $m_{i}$ in $M^{\prime}$. The $n-K$ remaining unmatched men in $U$ are given a partner from $Y$, and likewise, the $n-K$ remaining unmatched women in $W$ are given a partner from $X$. Clearly $M^{\prime}$ is a matching in $I$, and it is straightforward to verify that $M$ is stable.

Conversely suppose that $M^{\prime}$ is a stable matching in $I$. Then each of the $n-K$ women in $Y$ is matched with a man in $U$. Thus in $M^{\prime}$, exactly $K$ men of the form $m_{i}$ is matched with a woman of the form $w_{j}$; let $M$ contain the corresponding 
$\left\{m_{i}, w_{j}\right\}$ edges in $G$. Clearly $M$ is a matching in $G$ of size $K$, and the stability of $M^{\prime}$ implies that $M$ is maximal.

The above transformation can be modified to show that SMP-ILT remains NP-complete even if the ties occur in the preference lists of one sex only, any tie occurs at the tail of some person's preference list, and a tie is of length 2 (details are omitted). This restriction arises naturally in practice: for example, in the applicants-hospitals matching problem, although applicants might be able to rank hospitals in strict order, a large hospital may wish to rank only a subset of its applicants in strict order, expressing indifference among the remainder.

\section{Optimization Problems and Inapproximability}

Once the NP-completeness of a problem is proved, our next step is to try to find approximation algorithms for its optimization version. A goodness measure of an approximation algorithm $T$ of a minimization problem $P$ is defined as usual: let $x$ be an instance of the problem $P$ of size $N$. Also, let opt $(x)$ and $T(x)$ be the costs of an optimal solution and a solution obtained by the algorithm $T$, respectively. $T$ is said to be an $r(N)$-approximation algorithm if, for every $x, T(x) / o p t(x) \leq$ $r(N)$. If there exists a polynomial-time bounded $r(N)$-approximation algorithm for $P$, then we say that $P$ is approximable within $r(N)$.

There are several optimization problems that have only poor approximation algorithms. Among others, MAX-CLIQUE has received much attention recently because of its novel use of PCP. The latest result [5] says that this problem is not approximable within $N^{1-\epsilon}$ for any $\epsilon>0$ assuming $\mathrm{NP} \neq \mathrm{coRP}$, where $N$ denotes the number of vertices in a given graph. MIN-UN-3SAT is also hard, which requires, given a $3 \mathrm{CNF}$ formula, to find a truth assignment that minimizes the number of unsatisfied clauses. So MIN-UN-3SAT is basically the same problem as MAX-3SAT; only the cost function differs. It is shown that MIN-UN-3SAT is not approximable within $N^{1-\epsilon}$ for any $\epsilon>0$, where $N$ is the number of clauses in a given formula [7]. This result is obtained using a reduction from 3SAT, its NP-complete counterpart. The reduction makes many "copies" of an instance for 3SAT, which amplifies the gap of the cost between yes-instances and noinstances. We exploit this technique to show the inapproximability result for MIN-SMP-TIES. In doing so, a crucial point is how to "amplify" the gap, or how to "copy" the original instance so that the cost of solution will increase in a very different rate depending on the answer (yes/no) of the original instance. In the case of 3SAT, this is almost trivial since if the original formula is satisfiable then the number of unsatisfiable clauses is still zero for arbitrarily many copies. Our present case is not that easy.

Theorem 2. If $\mathrm{P} \neq \mathrm{NP}$, then MIN-SMP-TIES is not approximable within $N^{1-\epsilon}$ for any $\epsilon>0$.

Proof. Let $I$ be an instance of SMP-ILT obtained in the proof of Theorem 1. Let $K=9 l+3 n+t+3$, which is the number of men and also the number of women in $I$. We translate $I$ into an instance of MIN-SMP-TIES. Our target instance has $2 K^{c}$ men and the same number of women, where $c$ is some constant. First of all, we introduce the set $M_{U}$ of $K^{c}$ men $m_{0,1}, m_{0,2}, \cdots, m_{0, K^{c}}$ and $W_{U}$ of $K^{c}$ women $w_{0,1}, w_{0,2}, \cdots, w_{0, K^{c}}$. Next, we introduce another $K^{c-1}$ sets $M_{1}, M_{2}, \cdots, M_{K^{c-1}}$ of men, each of which contains exactly $K$ men. Similarly, we introduce $K^{c-1}$ sets $W_{1}, W_{2}, \cdots, W_{K^{c-1}}$ of women, each of which contains $K$ women. $M_{1}, M_{2}, \cdots, M_{K^{c-1}}$ can be considered to be "copies" of the set of men in $I$, namely, we associate each man in $M_{i}$ with each man in $I$, and each woman in $W_{i}$ with each woman in $I$, by one-to-one correspondence. Now we have $2 K^{c}$ men and $2 K^{c}$ women in total. 
Then we generate each person's preference list. Each man $m_{0, i}$ in $M_{U}$ writes the woman $w_{0, i}$ in $W_{U}$ at the top of his preference list, and then writes the other $2 K^{c}-1$ women arbitrarily. Each woman $w_{0, i}$ in $W_{U}$ writes the man $m_{0, i}$ in $M_{U}$ at the top of her preference list, and then writes the other $2 K^{c}-1$ men arbitrarily. It should be noted that in any stable matching, $m_{0, i}$ must be matched with $w_{0, i}$ since they write each other at the top of their preference lists.

Recall that, the men in $M_{i}$ play the same role as the men in $I$ and also the women in $W_{i}$ play the same role as the women in $I$. The list of a man $m$ (in $M_{i}$ ) is just the same as the list of the man $m^{\prime}$ in $I$ who corresponds to $m$ (but, of course, the women in the list are not the ones in $I$ but the corresponding women in $\left.W_{i}\right)$. We call these women, i.e., the ones who now exist in $m$ 's list, proper for $m$. Note that $m$ 's list is not yet complete, so we have to make it complete. First, we add $K^{c}$ women in $W_{U}$ to the list arbitrarily but in total order right after the proper women, and then finally we add the remaining women to the list arbitrarily and in total order. The women's lists are similar; namely, a list of each woman $w$ in $W_{i}$ is the same as the list of $w^{\prime}$ in $I$. We also call these men in the list proper for $w$. Then we add $K^{c}$ men in $M_{U}$ and we add the remaining men arbitrarily as before. Now we have an instance, $J$, of MIN-SMP-TIES. As shown in [4], there is always a stable matching for $J$ if we do not care its cost.

Thus the list of $m$ in $M_{i}$ includes proper women at the beginning, and then the long sequence of $w_{0, j}$ in $W_{U}$, and finally improper women. Hence, if $m$ is not matched with a proper woman, he has to "jump" the long sequence of $w_{0, j}$ 's and has to be matched with some improper woman. Formally, we can claim that the optimal cost for $J$ is at most $K^{c+1}$ when there is a stable matching for $I$ and is greater than $2 K^{2 c-1}$ otherwise. Hence if there were a polynomial-time approximation algorithm with approximation ratio as good as $\left(2 K^{2 c-1}\right) / K^{c+1}=$ $2 K^{c-2}$, then it could determine whether there is a stable matching for $I$ in polynomial time (recall that $c$ is a constant). Note that the size, $N$, of $J$ is $2 K^{c}$ and it follows that $2 K^{c-2}=\frac{2}{2^{1-\frac{2}{c}}} N^{1-\frac{2}{c}}>N^{1-\frac{2}{c}}$, which completes the proof.

\section{References}

1. D. Gale and L. S. Shapley, "College admissions and the stability of marriage," Amer. Math. Monthly, Vol.69, pp.9-15, 1962.

2. D. Gale and M. Sotomayor, "Some remarks on the stable matching problem," Discrete Applied Mathematics, Vol.11, pp.223-232, 1985.

3. M. R. Garey and D. S. Johnson, "Computers and Intractability, A Guide to The Theory of NP-Completeness," Freeman, San Francisco, 1979.

4. D. Gusfield and R. W. Irving, "The Stable Marriage Problem: Structure and Algorithms," MIT Press, Boston, MA, 1989.

5. J. Håstad, "Clique is hard to approximate within $n^{1-\epsilon}, "$ Proc. FOCS96, pp.627636, 1996

6. R. W. Irving, "Stable marriage and indifference," Discrete Applied Mathematics, Vol.48, pp.261-272, 1994.

7. T. Ohguro, "On the hardness of approximating minimization problems: in comparison with maximization classes," Technical Report of IEICE, COMP94-112 pp.8996, 1995.

8. E. Ronn, "NP-complete stable matching problems," J. Algorithms, Vol.11, pp.285304, 1990 .

9. M. Yannakakis and F. Gavril, "Edge dominating sets in graphs," SIAM J. Applied Mathematics, Vol.18, No.1, pp.364-372, 1980. 


\begin{tabular}{|c|c|c|c|c|c|c|c|}
\hline$m_{A, 1}$ & $w_{a, 1}$ & & & & & & \\
\hline$m_{A, 2}$ & $w_{a, 2}$ & & & & & & \\
\hline$m_{A, 3}$ & $w_{a, 3}$ & & & & & & \\
\hline$m_{A, 4}$ & $w_{a, 4}$ & & & & & & \\
\hline$m_{A, 5}$ & $w_{a, 5}$ & & & & & & \\
\hline$m_{B, 1,1}$ & $w_{d, 1,1}^{1}$ & $w_{d, 2,1}^{1}$ & $w_{d, 3,1}^{1}$ & & & & \\
\hline$m_{B, 1,2}$ & $w_{d, 1,1}^{0}$ & $w_{d, 2,1}^{0}$ & $w_{d, 3,1}^{0}$ & & & & \\
\hline$m_{B, 1,3}$ & $w_{d, 1,1}^{0}$ & $w_{d, 2,1}^{0}$ & $w_{d, 3,1}^{0}$ & & & & \\
\hline$m_{B, 2,1}$ & $w_{d, 1,2}^{1}$ & $w_{d, 3,2}^{1}$ & $w_{d, 4,2}^{1}$ & & & & \\
\hline$m_{B, 2,2}$ & $w_{d, 1,2}^{0}$ & $w_{d, 3,2}^{0}$ & $w_{d, 4,2}^{0}$ & & & & \\
\hline$m_{B, 2,3}$ & $w_{d, 1,2}^{0}$ & $w_{d, 3,2}^{0}$ & $w_{d, 4,2}^{0}$ & & & & \\
\hline$m_{C, 1}$ & $w_{a, 1}$ & $w_{a, 2}$ & $w_{a, 3}$ & $w_{a, 4}$ & $w_{a, 5}$ & $w_{b, 1}^{0}$ & $w_{b, 1}^{1}$ \\
\hline$m_{C, 2}$ & $w_{a, 1}$ & $w_{a, 2}$ & $w_{a, 3}$ & $w_{a, 4}$ & $w_{a, 5}$ & $w_{b, 2}^{0}$ & $w_{b, 2}^{1}$ \\
\hline$m_{C, 3}$ & $w_{a, 1}$ & $w_{a, 2}$ & $w_{a, 3}$ & $w_{a, 4}$ & $w_{a, 5}$ & $w_{b, 3}^{0}$ & $w_{b, 3}^{1}$ \\
\hline$m_{C, 4}$ & $w_{a, 1}$ & $w_{a, 2}$ & $w_{a, 3}$ & $w_{a, 4}$ & $w_{a, 5}$ & $w_{b, 4}^{0}$ & $w_{b, 4}^{1}$ \\
\hline$m_{D, 1}^{+}$ & $w_{a, 1}$ & $w_{c, 1}$ & $w_{d, 1,1}^{1}$ & $w_{d, 1,2}^{1}$ & $w_{b, 1}^{0}$ & & \\
\hline$m_{D, 1}^{-}$ & $w_{c, 1}$ & $w_{a, 2}$ & $w_{d, 1,1}^{0}$ & $w_{d, 1,2}^{0}$ & $w_{b, 1}^{1}$ & & \\
\hline$m_{D, 2}^{+}$ & $w_{a, 1}$ & $w_{c, 2}$ & $w_{d, 2,1}^{1}$ & $w_{a, 4}$ & $w_{b, 2}^{0}$ & & \\
\hline$m_{D, 2}^{-}$ & $w_{c, 2}$ & $w_{a, 2}$ & $w_{d, 2,1}^{0}$ & $w_{a, 4}$ & $w_{b, 2}^{1}$ & & \\
\hline$m_{D, 3}^{+}$ & $w_{a, 1}$ & $w_{c, 3}$ & $w_{d, 3,1}^{1}$ & $w_{d, 3,2}^{1}$ & $w_{b, 3}^{0}$ & & \\
\hline$m_{D, 3}^{-}$ & $w_{c, 3}$ & $w_{a, 2}$ & $w_{d, 3,1}^{0}$ & $w_{d, 3,2}^{0}$ & $w_{b, 3}^{1}$ & & \\
\hline$m_{D, 4}^{+}$ & $w_{a, 1}$ & $w_{c, 4}$ & $w_{d, 4,2}^{1}$ & $w_{a, 4}$ & $w_{b, 4}^{0}$ & & \\
\hline$m_{D, 4}^{-}$ & $w_{c, 4}$ & $w_{a, 2}$ & $w_{d, 4,2}^{0}$ & $w_{a, 4}$ & $w_{b, 4}^{1}$ & & \\
\hline$m_{E, 1,1}^{+}$ & $w_{a, 1}$ & $w_{e, 1,1}$ & $w_{d, 1,1}^{1}$ & & & & \\
\hline$m_{E, 1,1}^{-}$ & $w_{e, 1,1}$ & $w_{a, 2}$ & $w_{d, 1,1}^{0}$ & & & & \\
\hline$m_{E, 1,2}^{+}$ & $w_{a, 1}$ & $w_{e, 1,2}$ & $w_{a, 3}$ & $w_{d, 1,2}^{1}$ & & & \\
\hline$m_{E, 1,2}^{-}$ & $w_{e, 1,2}$ & $w_{a, 2}$ & $w_{a, 3}$ & $w_{d, 1,2}^{0}$ & & & \\
\hline$m_{E, 2,1}^{+}$ & $w_{a, 1}$ & $w_{e, 2,1}$ & $w_{d, 2,1}^{1}$ & & & & \\
\hline$m_{E, 2,1}^{-}$ & $w_{e, 2,1}$ & $w_{a, 2}$ & $w_{d, 2,1}^{0}$ & & & & \\
\hline$m_{E, 3,1}^{+}$ & $w_{a, 1}$ & $w_{e, 3,1}$ & $w_{d, 3,1}^{1}$ & & & & \\
\hline$m_{E, 3,1}^{-}$ & $w_{e, 3,1}$ & $w_{a, 2}$ & $w_{d, 3,1}^{0}$ & & & & \\
\hline$m_{E, 3,2}^{+}$ & $w_{a, 1}$ & $w_{e, 3,2}$ & $w_{a, 3}$ & $w_{d, 3,2}^{1}$ & & & \\
\hline$m_{E, 3,2}^{-}$ & $w_{e, 3,2}$ & $w_{a, 2}$ & $w_{a, 3}$ & $w_{d, 3,2}^{0}$ & & & \\
\hline$m_{E, 4,2}^{+}$ & $w_{a, 1}$ & $w_{e, 4,2}$ & $w_{d, 4,2}^{1}$ & & & & \\
\hline$m_{E, 4,2}^{-}$ & $w_{e, 4,2}$ & $w_{a, 2}$ & $w_{d, 4,2}^{0}$ & & & & \\
\hline
\end{tabular}

Table 2. Preference lists of men of $T\left(f_{0}\right)$ 